\title{
UNCONDITIONAL BASES OF REPRODUCING KERNELS IN HILBERT SPACES OF ENTIRE FUNCTIONS
}

\author{
K.P. ISAEV, R.S. YULMUKHAMETOV
}

\begin{abstract}
We consider the existence of unconditional bases of reproducing kernels in a functional Hilbert space of entire functions. It is proved that under certain conditions, unconditional bases of reproducing kernels do not exist. It is shown that in particular spaces, some known theorems on the absence of unconditional bases are the consequences of these results.
\end{abstract}

Keywords: Hilbert spaces, entire functions, reproducing kernels, unconditional bases.

\section{INTRODUCTION}

Let $H$ be a functional Hilbert space of entire functions. That is, the functionals $\delta_{z}: f \rightarrow f(z)$ are continuous for each $z \in \mathbb{C}$. Then each functional $\delta_{z}$ is generated by an element $k_{z}(\lambda) \in H$ in the sense $\delta_{z}(f)=\left(f(\lambda), k_{z}(\lambda)\right)$. The function $k(\lambda, z)=k_{z}(\lambda)$ is called reproducing kernel. The function $\left\|k_{z}(\lambda)\right\|_{H}=(k(z, z))^{\frac{1}{2}}$ called Bergman function of space $H$. The main properties of reproducing kernels in Hilbert spaces are presented in work [1]. Denote $K(z)=\left\|k_{z}(\lambda)\right\|_{H}^{2}$. In what follows, we make additional assumptions for space $H$,

$$
K(z)>0, z \in \mathbb{C}
$$

if $f \in H$ and $z_{0}$ is a zero of function $f(z)$, then

$$
\frac{f(z)}{z-z_{0}} \in H .
$$

The system of elements $e_{k}, k=1,2, \ldots$, in a Hilbert space is called unconditional basis (cf. [2]) if it is complete and there exist numbers $c, C>0$, such that for each set of numbers $c_{1}, c_{2}$, $\ldots, c_{n}$ the relation

$$
c \sum_{k=1}^{n}\left|c_{k}\right|^{2}\left\|e_{k}\right\|^{2} \leq\left\|\sum_{k=1}^{n} c_{k} e_{k}\right\|^{2} \leq C \sum_{k=1}^{n}\left|c_{k}\right|^{2}\left\|e_{k}\right\|^{2}
$$

holds true. It is known (cf. [3], [4]), that if a system $e_{k}, k=1,2, \ldots$, is an unconditional basis, then each element of space $H$ is uniquely represented by the series

$$
x=\sum_{k=1}^{\infty} x_{k} e_{k}
$$

and

$$
c \sum_{k=1}^{\infty}\left|x_{k}\right|^{2}|| e_{k}\left\|^{2} \leq\right\| x\left\|^{2} \leq C \sum_{k=1}^{\infty}\left|x_{k}\right|^{2}\right\| e_{k} \|^{2} .
$$

K.P. Isaev, R.S. Yulmukhametov, Unconditional Bases of Reproducing Kernels in Hilbert SPACES OF ENTIRE FUNCTIONS.

(C) Isaev K.P., Yulmukhametov R.S. 2013.

The work is supported by FTP "Scientific and pedagogical staff of innovative Russia", contract no. 14.B37.21.0358.

Submitted December 12, 2012. 
Given a sequence of complex numbers $\left\{z_{j}\right\}_{j=1}^{\infty} \subset \mathbb{C}$, let us consider the system $\left\{k\left(\lambda, z_{j}\right)\right\}_{j=1}^{\infty}$. We shall be interested in the question on the conditions for the sequence $\left\{z_{j}\right\}_{j=1}^{\infty}$ guaranteeing that the corresponding system of reproducing kernels is an unconditional basis in space $H$.

In the dual formulation, the problem on unconditional bases of reproducing kernels in Hilbert space of entire functions becomes an interpolation problem. In some special cases, by FourierLaplace transformation, the problem becomes equivalent to that on unconditional bases formed by exponentials. The basic examples here are classical Fourier series in $L_{2}(-\pi, \pi)$, interpolation theorem in Paley-Wiener space (Kotelnikov theorem).

In weighted spaces of entire functions

$$
F_{\varphi}=\left\{f \in H(\mathbb{C}):\|f\|^{2}:=\int_{\mathbb{C}}|f(z)|^{2} e^{-2 \varphi(z)} d m(z)<\infty\right\}
$$

where $\varphi(z)$ is a subharmonic function, $d m(z)$ Lebesgue measure, the problem on unconditional bases of reproducing kernels was treated in many works. Among recent ones, we mention [5], $[6],[8],[9]$.

In the present work, in Section 3, we prove that under certain conditions there are unconditional bases in space $H$. In Section we demonstrate how the proven theorems imply known theorems for particular spaces.

\section{Formulation of MAin Results}

In Section 3 we shall prove

Theorem 1. If a system $\left\{k\left(\lambda, z_{j}\right)\right\}_{j=1}^{\infty}$ is an unconditional basis in space $H$, there exists an entire function $L$ with simple zeroes at the points $z_{j}, j=1,2, \ldots$, satisfying relation

$$
\frac{1}{P} K(z) \leq \sum_{i=j}^{\infty} \frac{|L(z)|^{2} K\left(z_{j}\right)}{\left|L^{\prime}\left(z_{j}\right)\right|^{2}\left|z-z_{j}\right|^{2}} \leq P K(z), z \in \mathbb{C},
$$

where $P$ is a some positive constant.

We introduce one more characteristic for the continuous on the plane functions $u$ measuring the deviation of a given function from harmonic ones. For a continuous function $u, z \in \mathbb{C}$ and a positive number $p$, by $\tau(u, z, p)$ we denote the supremum of all $r>0$ such that

$$
\inf \left\{\sup _{w \in B(z, r)}|u(w)-h(w)|, h \text { is harmonic in } B(z, r)\right\} \leq p .
$$

Here $B(z, r)$ is a circle centered at $z$ of radius $r$. Immediately from the definition it follows that if $\tau\left(u, z_{0}, p\right)=\infty$ for some point $z_{0}$, then $\tau(u, z, p) \equiv \infty$. We have the following statement.

Lemma 1. If for a function $u$, the characteristics $\tau(u, z, p)$ is finite, then function $\tau(z)=\tau(u, z, p)$ satisfies Lipshitz condition,

$$
\left|\tau\left(z_{1}\right)-\tau\left(z_{2}\right)\right| \leq\left|z_{1}-z_{2}\right|
$$

for all $z_{1}$ and $z_{2}$.

A simple proof of this lemma can be found in [7].

In work [10], it was shown (Lemma 1.1) that in the case $u$ is a continuous subharmonic function, the quantity $\tau=\tau(u, \lambda, p)$ is well-defined by the condition: if $H(z)$ is the smallest harmonic majorant of function $u$ in the circle $B(\lambda, \tau)$, then

$$
\max _{z \in \bar{B}(\lambda, \tau)}(H(z)-u(z))=2 p
$$

The function

$$
\ln K(z)=2 \sup _{\|F\| \leq 1} \ln |F(z)|
$$


is subharmonic and continuous on the whole plane (we suppose that $K(z)>0$ ). In what follows, by $\tau(z)$ we denote the function $\tau(\ln K(w), z, \ln (5 P))$, where $P$ is the constant in (5). Hence,

$$
\inf \left\{\sup _{z \in \bar{B}(\lambda, \tau(\lambda))}|\ln K(z)-h(z)|, \mathrm{h} \text { is harmonic in } B(z, \tau(z))\right\}=\ln (5 P) .
$$

The next theorem was proven in [7] (see Theorem 1).

Theorem 2. Let $L(z)$ be an entire function with simple zeroes $z_{i}, i=1,2, \ldots$ satisfying the double-sided estimate

$$
\frac{1}{P} K(z) \leq \sum_{i=1}^{\infty} \frac{|L(z)|^{2} K\left(z_{i}\right)}{\left|L^{\prime}\left(z_{i}\right)\right|^{2}\left|z-z_{i}\right|^{2}} \leq P K(z)
$$

with some $P$. Then

1) In each circle $B(z, 2 \tau(z))$ there exists at least one zero $z_{i}$ of function $L$.

2) For each $i, j, i \neq j$, inequality

$$
\left|z_{i}-z_{j}\right| \geq \frac{\max \left(\tau\left(z_{i}\right), \tau\left(z_{j}\right)\right)}{10 P^{\frac{3}{2}}}
$$

holds true.

3) For each $i$ in each circle $B\left(z_{i}, \frac{\tau\left(z_{i}\right)}{20 P^{\frac{3}{2}}}\right)$ the relation

$$
\frac{1}{5^{6} P^{8}} K(z) \leq \frac{K\left(z_{i}\right)|L(z)|^{2}}{\left|L^{\prime}\left(z_{i}\right)\right|^{2}\left|z-z_{i}\right|^{2}} \leq P K(z)
$$

is valid.

In Section 3 we shall prove Theorems 3,4.

Theorem 3. Let $z_{i}, i=1,2, \ldots$, be the zeroes of function $L(z)$ satisfying the hypothesis of the previous theorem. Then for each finite set of zeroes $B$ containing at least two zeroes, there exists an index $n$ such that

$$
\sum_{z_{i} \in B, i \neq n} \frac{\tau^{2}\left(z_{i}\right)}{\left|z_{i}-z_{n}\right|^{2}} \leq(4 P)^{12} .
$$

Corollary. Let $z_{i}, i=1,2, \ldots$, be the zeroes of function $L(z)$ satisfying the hypothesis of the previous theorem and $b=\frac{1}{20 P^{\frac{3}{2}}}$. Then for each finite set of zeroes $B$ containing at least two zeroes, there exists an index $n$ such that

$$
\sum_{z_{i} \in B, i \neq n} \int_{B\left(z_{i}, b \tau\left(z_{i}\right)\right)} \frac{d m(z)}{\left|z-z_{n}\right|^{2}} \leq 4^{10} P^{9} .
$$

Theorem 4. Let $H$ be the functional Hilbert space of entire functions satisfying conditions (1) and (2). Suppose that for each positive number $p$ there exists a number $\delta=\delta(p)>0$ such that the function $\tau(z)=\tau(\ln K(\lambda), z, p)$ satisfies the condition

$$
\inf _{z \in B(\lambda, 2 \tau(\lambda))} \tau(z) \geq \delta \tau(\lambda)
$$

for all $\lambda \in \mathbb{C}$ and $\tau(z)=o(|z|)$ as $|z| \longrightarrow \infty$. Then there exist no unconditional bases of reproducing kernels in space $H$.

A slight modification of the proof of this theorem leads one to essentially weaker assumptions.

Theorem 5. Let $H$ be the functional Hilbert space of entire functions satisfying conditions (1) and (2). Suppose for each positive number $p$ there exists a number $\delta=\delta(p)>0$ 
and a sequence of circles $B\left(\zeta_{j}, R_{j}\right)$ (depending on number $p$ ) such that the function $\tau(z)=$ $\tau(\ln K(\lambda), z, p)$ satisfies the condition

$$
\inf _{z \in B(\lambda, 2 \tau(\lambda))} \tau(z) \geq \delta \tau(\lambda)
$$

for all $\lambda \in B\left(\zeta_{j}, R_{j}\right)$. And moreover,

$$
\max _{z \in \bar{B}\left(\zeta_{j}, R_{j}\right)} \tau(z)=o\left(R_{j}\right), j \longrightarrow \infty .
$$

Then there exist no unconditional bases of reproducing kernels in space $H$.

\section{Proof of MAin STATEMENTS}

Proof of Theorem 1. Let $k(\lambda, z)=\sum_{j=1}^{\infty} c_{j}(z) k\left(\lambda, z_{j}\right), \lambda \in \mathbb{C}$. We observe that $c_{j}\left(z_{k}\right)=\delta_{j}^{k}$, where $\delta_{j}^{k}$ is the Kronecker delta. Let $\left\{S_{j}(\lambda)\right\}_{j=1}^{\infty} \subset H$ be the biorthogonal system for the system $k\left(\lambda, z_{j}\right)$, i.e., $\left(S_{j}(\lambda), k\left(\lambda, z_{k}\right)\right)=\delta_{j}^{k}$. Then

$$
\left(k(\lambda, z), S_{j}(\lambda)\right)=\sum_{k=1}^{\infty} c_{k}(z)\left(k\left(\lambda, z_{k}\right), S_{j}(\lambda)\right),
$$

hence, $\overline{S_{j}(z)}=c_{j}(z)$. This is why $S_{j}\left(z_{k}\right)=\delta_{k}^{j}$. We note that $z_{k}, k \neq j$ are simple zeroes of function $S_{j}(z)$. Indeed, if for some $m \neq j$ the quantity $S_{j}^{\prime}\left(z_{m}\right)$ was equal to zero, then the function $\left(z_{j}-z_{m}\right) S_{j}(z) /\left(z-z_{m}\right)$ belong to $H$ due to $(2)$ would vanish at the points $z_{k}, k \neq j$ and would be equal to one at the point $z_{j}$, i.e., at all the points $z_{k}, k=1,2,3, \ldots$, it would coincide with function $S_{j}(z)$. But due to the completeness of the system $\left\{k\left(\lambda, z_{k}\right)\right\}$ in space $H$, the system of points $z_{k}, k=1,2,3, \ldots$, is the uniqueness set for space $H$. Thus, functions $\left(z_{j}-z_{m}\right) S_{j}(z) /\left(z-z_{m}\right)$ and $S_{j}(z)$ would have to identically coincide.

The function $L(z)=S_{1}(z)\left(z-z_{1}\right)$ is an entire one. The points $z_{k}, k=1,2, \ldots$, are its simple zeroes. It is obvious that

As $j>1$,

$$
S_{1}(z)=\frac{L(z)}{L^{\prime}\left(z_{1}\right)\left(z-z_{1}\right)} .
$$

$$
\frac{L(z)}{L^{\prime}\left(z_{j}\right)\left(z-z_{j}\right)}=\frac{S_{1}(z)}{L^{\prime}\left(z_{j}\right)}+\frac{S_{1}(z)\left(z_{j}-z_{1}\right)}{L^{\prime}\left(z_{j}\right)\left(z-z_{j}\right)} \in H
$$

and it coincides with $S_{j}(z)$ at all the points $z_{k}, k=1,2, \ldots$. Again due to the completeness of the system $\left\{k\left(\lambda, z_{k}\right)\right\}$,

$$
S_{j}(z)=\frac{L(z)}{L^{\prime}\left(z_{j}\right)\left(z-z_{j}\right)}, j=2,3, \ldots
$$

this is why

$$
k(\lambda, z)=\sum_{i=1}^{\infty} \frac{\overline{L(z)}}{\overline{L^{\prime}\left(z_{i}\right)\left(z-z_{i}\right)}} k\left(\lambda, z_{i}\right), \lambda \in \mathbb{C} .
$$

Employing (3), we arrive at the statement of the theorem. Above arguments mean that the system

$$
\frac{L(z)}{L^{\prime}\left(z_{j}\right)\left(z-z_{j}\right)}, j=1,2, \ldots,
$$

is the biorthogonal one for the original system of reproducing kernels. The proof is complete.

Proof of Theorem 3. By the hypothesis of the theorem, the estimate

$$
\sum_{z_{i} \in B} \frac{K\left(z_{i}\right)|L(z)|^{2}}{\left|L^{\prime}\left(z_{i}\right)\right|^{2}\left|z-z_{i}\right|^{2}} \leq P K(z)
$$


holds true for each $z$. Since set $B$ is finite, there exists an index $n$ such that

$$
\frac{K\left(z_{n}\right)}{\left|L^{\prime}\left(z_{n}\right)\right|^{2} \tau^{2}\left(z_{n}\right)}=\min _{z_{i} \in B}\left(\frac{K\left(z_{i}\right)}{\left|L^{\prime}\left(z_{i}\right)\right|^{2} \tau^{2}\left(z_{i}\right)}\right) .
$$

By Item 3 of Theorem 2 , for the points $z$ on the boundary of the circle $B\left(z_{n}, \frac{1}{20 P^{\frac{3}{2}}} \tau\left(z_{n}\right)\right)$ the estimate

is valid, or

$$
\frac{1}{5^{6} P^{8}} K(z) \leq 20^{2} P^{3} \frac{K\left(z_{n}\right)|L(z)|^{2}}{\left|L^{\prime}\left(z_{n}\right)\right|^{2} \tau^{2}\left(z_{n}\right)}
$$

Together with estimate (7) it implies

$$
\frac{K(z)}{|L(z)|^{2}} \leq 4^{2} 5^{8} P^{11} \frac{K\left(z_{n}\right)}{\left|L^{\prime}\left(z_{n}\right)\right|^{2} \tau^{2}\left(z_{n}\right)} .
$$

$$
4^{2} 5^{8} P^{11} \frac{K\left(z_{n}\right)}{\left|L^{\prime}\left(z_{n}\right)\right|^{2} \tau^{2}\left(z_{n}\right)} \geq \frac{1}{P} \sum_{z_{i} \in B} \frac{K\left(z_{i}\right)}{\left|L^{\prime}\left(z_{i}\right)\right|^{2}\left|z-z_{i}\right|^{2}} .
$$

Therefore, for the points $z$ on the boundary of the circle $B\left(z_{n}, \frac{1}{20 P^{\frac{3}{2}}} \tau\left(z_{n}\right)\right)$,

$$
4^{2} 5^{8} P^{12} \frac{K\left(z_{n}\right)}{\left|L^{\prime}\left(z_{n}\right)\right|^{2} \tau^{2}\left(z_{n}\right)} \geq \sum_{z_{i} \in B} \frac{K\left(z_{i}\right)}{\left|L^{\prime}\left(z_{i}\right)\right|^{2} \tau^{2}\left(z_{i}\right)} \cdot \frac{\tau^{2}\left(z_{i}\right)}{\left|z-z_{i}\right|^{2}} .
$$

Taking into consideration the choice of index $n$, for the points $z$ on the boundary of $B\left(z_{n}, \frac{1}{20 P^{\frac{3}{2}}} \tau\left(z_{n}\right)\right)$, we have

$$
4^{2} 5^{8} P^{12} \frac{K\left(z_{n}\right)}{\left|L^{\prime}\left(z_{n}\right)\right|^{2} \tau^{2}\left(z_{n}\right)} \geq \frac{K\left(z_{n}\right)}{\left|L^{\prime}\left(z_{n}\right)\right|^{2} \tau^{2}\left(z_{n}\right)} \sum_{z_{i} \in B} \frac{\tau^{2}\left(z_{i}\right)}{\left|z-z_{i}\right|^{2}},
$$

or

$$
\sum_{z_{i} \in B} \frac{\tau^{2}\left(z_{i}\right)}{\left|z-z_{i}\right|^{2}} \leq 4^{2} 5^{8} P^{12}
$$

By Item 2 of Theorem 2, for the mentioned points $z$ as $i \neq n$, the estimate

$$
\left|z-z_{i}\right| \leq\left|z-z_{n}\right|+\left|z_{n}-z_{i}\right|=\frac{\tau\left(z_{n}\right)}{20 P^{\frac{3}{2}}}+\left|z_{n}-z_{i}\right| \leq \frac{3}{2}\left|z_{n}-z_{i}\right|
$$

holds true, and hence, the desired estimate is implied by (8),

$$
\sum_{z_{i} \in B, i \neq n} \frac{\tau^{2}\left(z_{i}\right)}{\left|z_{n}-z_{i}\right|^{2}} \leq(4 P)^{12} .
$$

The proof is complete.

Proof of Corollary of Theorem 3. Since for the points $z \in B\left(z_{i}, b \tau\left(z_{i}\right)\right)$ we have

$$
\left|z-z_{n}\right| \geq\left|z_{i}-z_{n}\right|-\left|z-z_{i}\right| \geq \frac{1}{2}\left|z_{i}-z_{n}\right|
$$

then

It yields

$$
\int_{B\left(z_{i}, b \tau\left(z_{i}\right)\right)} \frac{d m(z)}{\left|z-z_{n}\right|^{2}} \leq \frac{4 \pi b^{2} \tau^{2}\left(z_{i}\right)}{\left|z_{i}-z_{n}\right|^{2}}
$$

$$
\sum_{z_{i} \in B, i \neq n} \int_{B\left(z_{i}, b \tau\left(z_{i}\right)\right)} \frac{d m(z)}{\left|z-z_{n}\right|^{2}} \leq 4 \pi b^{2}(4 P)^{12}=\frac{4 \pi}{400 P^{3}}(4 P)^{12} \leq 4^{10} P^{9} .
$$

Proof of Theorem 4. We shall make use of the following statement (see [11], p. 216). 
Lemma (On cover by balls). Suppose a set $A \subset \mathbb{R}^{p}$ is covered by balls so that each point $x \in A$ is the center of some ball $S(x)$ of radius $r(x)$. If $\sup _{x \in A} r(x)<\infty$, then from system $\{S(x)\}$, it is possible to select at most countable subsystem $\left\{S\left(x_{k}\right)\right\}$ covering set $A$ and having the multiplicity not exceeding some number $N(p)$ depending only on the space dimension.

It is easy to check that $N(2)=6$.

We prove theorem by contradiction. Assume the hypothesis of the theorem holds true, but in space $H$ there exists an unconditional basis of reproducing kernels $\left\{k\left(\lambda, z_{i}\right)\right\}$. Then Theorems 1,2, and 3 hold true. We let $p=\ln (5 P)$ and $\tau(z)=\tau(\ln K(\lambda), z, \ln (5 P))$.

We choose an arbitrary $\varepsilon>0$ and suppose a number $R$ is large enough to satisfy

$$
\max _{|z| \leq R} \tau(z) \leq \varepsilon R .
$$

Such $R$ exists due to the condition for $\tau(z)$. Indeed, there exists $R^{\prime}$ such that as $|z| \geq R^{\prime}$, the inequality $\tau(z)<\varepsilon|z|$ holds. If we take $R>\frac{2 R^{\prime}}{\varepsilon}$, then as $|z| \in\left[\frac{\varepsilon}{2} R ; R\right]$, we have $\tau(z)<\varepsilon|z| \leq$ $\varepsilon R$. By Lemma 1, the relation $\tau(z) \leq \tau(0)+|z|$ is valid and thus, if $|z| \in\left[\tau(0) ; \frac{\varepsilon}{2} R\right]$, then $\tau(z) \leq 2|z| \leq \varepsilon R$. Finally, choosing $R$ greater that $\frac{1}{\varepsilon} \max _{|z| \leq \tau(0)} \tau(z)$, we get relation (9).

Consider the system of circles $B(\lambda, 2 \tau(\lambda)), \lambda \in B(0, R)$. By Item 1 of Theorem 2, each of these circles contains at least one of $z_{i}$ and these circles cover $B(0, R)$. By lemma on cover by balls, there exists at most countable set of circles $B_{n}=B\left(\lambda_{n}, 2 \tau\left(\lambda_{n}\right)\right)$ covering $B(0, R)$ so that each point of the latter circle belongs to at most $N(2)=6$ circles of the cover. In each of the circles $B_{n}$, we choose one $z_{i(n)}$. At that, some of $z_{i(n)}$ can be chosen several times, but by the properties of subcover, the multiplicity of each index is at most six. We renumber the selected indices associating with them the index of the circle in which the index was chosen. We obtain the set of the numbers $\left\{w_{n}\right\}$, where each number appears at most six times. We then apply Theorem 3 to this set. There exists a number $m$ such that, with the multiplicity taken into account, the estimate

$$
\sum_{w_{n} \neq w_{m}} \frac{\tau^{2}\left(w_{n}\right)}{\left|w_{n}-w_{m}\right|^{2}} \leq 6(4 P)^{12}
$$

holds true.

In our notations $w_{n} \in B_{n}=B_{n}\left(\lambda_{n}, 2 \tau\left(\lambda_{n}\right)\right)$. We then consider $n$ such that $w_{m} \notin B_{n}^{\prime}=B_{n}\left(\lambda_{n}, 3 \tau\left(\lambda_{n}\right)\right)$. Then for each $w \in B_{n}$ we have $\left|w-w_{m}\right| \geq \tau\left(\lambda_{n}\right)$. Moreover,

$$
\left|w_{n}-w_{m}\right| \leqslant\left|w_{n}-w\right|+\left|w-w_{m}\right| \leq 4 \tau\left(\lambda_{n}\right)+\left|w-w_{m}\right| \leq 5\left|w-w_{m}\right|,
$$

or

$$
\frac{1}{\left|w-w_{m}\right|^{2}} \leq \frac{25}{\left|w_{n}-w_{m}\right|^{2}}, w \in B_{n}, w_{m} \notin B_{n}^{\prime} .
$$

Integrating this inequality over circle $B_{n}$, we obtain

$$
\int_{B_{n}} \frac{d m(w)}{\left|w-w_{m}\right|^{2}} \leq \frac{100 \pi \tau^{2}\left(\lambda_{n}\right)}{\left|w_{n}-w_{m}\right|^{2}}, w_{m} \notin B_{n}^{\prime} .
$$

Since $w_{n} \in B\left(\lambda_{n}, 2 \tau\left(\lambda_{n}\right)\right)$, by condition (6), $\tau^{2}\left(w_{n}\right) \geq \delta^{2} \tau^{2}\left(\lambda_{n}\right)$. Hence, the latter estimate and (10) imply

$$
\sum_{w_{n} \neq w_{m} \notin B_{n}^{\prime}} \int_{B_{n}} \frac{d m(w)}{\left|w-w_{m}\right|^{2}} \leq \frac{100 \pi}{\delta^{2}} \sum_{w_{n} \neq w_{m} \notin B_{n}^{\prime}} \frac{\tau^{2}\left(w_{n}\right)}{\left|w_{n}-w_{m}\right|^{2}} \leq \frac{600(4 P)^{12}}{\delta^{2}}:=C .
$$

If index $n$ is so that $w_{m} \in B_{n}^{\prime}$, then for each $w \in B_{n}$ we have

$$
\left|w-w_{m}\right| \leq\left|w-\lambda_{n}\right|+\left|w_{m}-\lambda_{n}\right| \leq 2 \tau\left(\lambda_{n}\right)+3 \tau\left(\lambda_{n}\right)=5 \tau\left(\lambda_{n}\right) .
$$

By the choice of number $R$, we have $\left|w-w_{m}\right| \leq 5 \varepsilon R$, i.e., circles $B_{n}$ lie inside the circle $B\left(w_{m}, 5 \varepsilon R\right)$. It means that the circles of the cover, whose indices are involved in the summation 
in (11), cover the set $C(R)=B(0, R) \backslash B\left(w_{m}, 5 \varepsilon R\right)$. Therefore,

$$
\int_{C(R)} \frac{d m(w)}{\left|w-w_{m}\right|^{2}} \leq C .
$$

We make the change of variables $w=R \zeta, w_{m}=R \zeta_{m}, \zeta_{m} \in B(0,1+2 \varepsilon)$ to obtain

$$
\int_{B(0,1) \backslash B\left(\zeta_{m}, 5 \varepsilon\right)} \frac{d m(\zeta)}{\left|\zeta-\zeta_{m}\right|^{2}} \leq C
$$

Number $\varepsilon>0$ was chosen arbitrary, and tending $\varepsilon$ to zero, we obtain the contradiction. The proof of Theorem 4 is complete.

\section{Weighted Hilbert SPACES of EntiRe FUnCtions}

In this section we consider more specific Hilbert spaces.

First we prove a statement allowing, under some assumptions for a subharmonic function $u$, to relate the asymptotic behavior of characteristics with the behavior of the Laplacian of function $u$.

Lemma 2. Suppose a subharmonic on the plane function $u$ is twice continuously differentiable and for each number $p>0$, there exists a number $b=b(p) \geq 1$ such that the condition

$$
\frac{1}{b} \leq \frac{\Delta u(w)}{\Delta u(z)} \leq b, w \in B\left(z, \sqrt{8 p b}(\Delta u(z))^{-\frac{1}{2}}\right)
$$

holds true. Then the estimates

$$
\sqrt{\frac{8 p}{b \Delta u(z)}} \leq \tau(u, z, p) \leq \sqrt{\frac{8 p b}{\Delta u(z)}}
$$

are valid.

Proof. For the sake of brevity, we introduce the notation $\rho(z)=(\Delta u(z))^{-\frac{1}{2}}$. As it was observed above, quantity $\tau=\tau(u, z, p)$ can be determined by the condition

$$
\max _{w \in \bar{B}(z, \tau)}\left(H_{u}(w)-u(w)\right)=2 p
$$

where $H_{u}$ is the minimal harmonic majorant for function $u$ in circle $B(z, \tau)$. By Green formula,

$$
h_{u}(w)-u(w)=\int_{B(z, r)} G(w, \zeta) d \mu_{u}(\zeta),
$$

where $h_{u}$ is the minimal harmonic majorant for function $u$ in circle $B(z, r), G(w, \zeta)$ is the Green function for this circle and $\mu_{u}$ is the associated measure of function $u$. In our case $2 \pi d \mu_{u}(\zeta)=\Delta u(\zeta) d m(\zeta)$, hence, quantity $\tau$ can be determined by the condition

$$
\max _{w \in \bar{B}(z, \tau)} \int_{B(z, \tau)} G(w, \zeta) \frac{\Delta u(\zeta)}{2 \pi} d m(\zeta)=2 p .
$$

If we let $r=\sqrt{\frac{8 p}{b}} \rho(z)\left(r \leq \sqrt{8 p b}(\Delta u(z))^{-\frac{1}{2}}\right)$, then

$$
\max _{w \in \bar{B}(z, r)} \int_{B(z, r)} G(w, \zeta) \frac{\Delta u(\zeta)}{2 \pi} d m(\zeta) \leq b \Delta u(z) \max _{w \in \bar{B}(z, r)} \int_{B(z, r)} G(w, \zeta) \frac{d m(\zeta)}{2 \pi} .
$$

The function $v(w)=|w-z|^{2}$ is subharmonic, its associated measure is identically equal to $\frac{2}{\pi}$, and the minimal harmonic majorant in circle $B(z, r)$ is identically equal to $r^{2}$. Thus,

$$
\max _{w \in \bar{B}(z, r)} \int_{B(z, r)} G(w, \zeta) \frac{d m(\zeta)}{2 \pi}=\frac{1}{4} \max _{w \in \bar{B}(z, r)}\left(r^{2}-|w-z|^{2}\right)=\frac{r^{2}}{4} .
$$


Hence,

$$
\max _{w \in \bar{B}(z, r)} \int_{B(z, r)} G(w, \zeta) \frac{\Delta u(\zeta)}{2 \pi} d m(\zeta) \leq \frac{b r^{2}}{4} \Delta u(z),
$$

and under the hypothesis of the theorem,

$$
\max _{w \in \bar{B}(z, r)} \int_{B(z, r)} G(w, \zeta) \frac{\Delta u(\zeta)}{2 \pi} d m(\zeta) \leq \frac{b r^{2}}{4} \Delta u(z)=2 p .
$$

It implies the lower bound for $\tau=\tau(u, z, p)$.

In the same way, taking $r=\sqrt{8 p b} \rho(z)$, we get the estimate

$$
\begin{gathered}
\max _{w \in \bar{B}(z, r)} \int_{B(z, r)} G(w, \zeta) \frac{\Delta u(\zeta)}{2 \pi} \\
d m(\zeta) \geq \frac{\Delta u(z)}{b} \max _{w \in \bar{B}(z, r)} \int_{B(z, r)} G(w, \zeta) \frac{d m(\zeta)}{2 \pi} \geq \\
\geq \frac{\Delta u(z)}{b} \cdot \frac{r^{2}}{4}=2 p .
\end{gathered}
$$

It yields the upper estimate for $\tau=\tau(u, z, p)$.

Let us show that Theorems 4 and 5 allow us to describe the situations of absence of unconditional bases in various spaces.

A) It was shown in work [9] that if $\varphi(z)=\varphi(|z|)$ is a subharmonic twice differentiable on the plane function and $\rho(z)=(\Delta \varphi(z))^{-\frac{1}{2}}$ obeys the conditions

$$
0<\inf _{r>0} \rho(r) \text { and } \rho(r)=o(r), r \rightarrow \infty,
$$

and also

$$
\rho(r+\rho(r))=(1+o(1)) \rho(r), \quad r \rightarrow \infty \text { and } \rho(2 r) \asymp \rho(r), \quad r>0,
$$

then in space $F_{\varphi}(4)$ there exist no unconditional bases of reproducing kernels.

The condition $\rho(2 r) \asymp \rho(r), r>0$, means that there exist constants $c, C>0$ such that

$$
c<\frac{\rho(2 r)}{\rho(r)}<C
$$

for all $r>0$.

Conditions (14) mean that $\varphi(x)$ increases faster that $(\ln x)^{2}$ and not faster that $x^{2}$ as $x \rightarrow \infty$.

Validity of (15) implies that for $\varphi(z)$, condition (12) of Lemma 4.1 holds true, and thus, estimates (13) are valid. Then conditions (14) yield the estimates

$$
\sqrt{\frac{8 p}{b \Delta \varphi(z)}} \leq \tau(\ln K(\lambda), z, p) \leq \sqrt{\frac{8 p b}{\Delta \varphi(z)}} .
$$

Thus, the hypothesis of Theorem 4 hold true that means the absence of unconditional bases of reproducing kernels.

B) Consider Smirnov space $E_{2}(D)$ and Bergman space $B_{2}(D)$, where $D$ is a bounded convex domain in the complex plane.

Space $E_{2}(D)$ is the completion of the space of polynomials w.r.t. the norm

$$
\|p\|^{2}=\int_{\partial D}|p(z)|^{2} d s(z)
$$

where $d s(z)$ is the differential of the boundary of $D$.

Space $B_{2}(D)$ comprises the functions analytic in $D$ and square integrable w.r.t. the planar Lebesgue measure,

$$
B_{2}(D)=\left\{f \in H(D): \int_{D}|f(z)|^{2} d m(z)<\infty\right\}
$$

The system $\left\{e^{\lambda z}, \lambda \in \mathbb{C}\right\}$ is complete in these spaces. This fact allows us to describe the adjoint spaces $E_{2}^{*}(D)$ and $B_{2}^{*}(D)$ in terms of Fourier-Laplace transformation. To each linear 
continuous functional $S$ in these spaces, we associated an entire function $\widehat{S}(\lambda)=S\left(e^{\lambda z}\right), \lambda \in \mathbb{C}$ called Fourier-Laplace transform of functional $S$.

It is shown in work [12] that Fourier-Laplace transform is an isomorphism between space $E_{2}^{*}(D)$ and the Hilbert space of entire functions $\widehat{E}_{2}(D)$ with the norm

$$
\|F\|^{2}=\int_{0}^{\infty} \int_{0}^{2 \pi} \frac{\left|F\left(r e^{i \varphi}\right)\right|^{2}}{K\left(r e^{i \varphi}\right)} d \Delta(\varphi) d r
$$

where

$$
\begin{gathered}
\Delta(\varphi)=\int_{0}^{\varphi} h(\alpha) d \alpha+h^{\prime}(\varphi), \quad h(\varphi)=\max _{z \in \bar{D}} R e z e^{i \varphi}, \\
K(\lambda)=\left\|e^{\lambda z}\right\|_{E_{2}(D)}^{2}=\int_{\partial D}\left|e^{\lambda z}\right|^{2} d s(z) .
\end{gathered}
$$

In work [13], there was that space $B_{2}^{*}(D)$ is isomorphic to the Hilbert space of entire functions $\widehat{B}_{2}(D)$ with the norm defined by formula (16), where

$$
K(\lambda)=\left\|e^{\lambda z}\right\|_{B_{2}(D)}^{2}=\int_{D}\left|e^{\lambda z}\right|^{2} d m(z) .
$$

Thanks to this fact, the problems on unconditional bases of exponentials $\left\{e^{\lambda_{k} z}\right\}_{k=1}^{\infty}$ in spaces $E_{2}(D)$ and $B_{2}(D)$ happen to be equivalent to the problems on unconditional bases of reproducing kernels in the adjoint spaces of entire functions.

It was shown in work [7] that if the boundary of a domain contains a point with a finite nonzero curvature, there exist no unconditional bases in Bergman space. This can be obtained by Theorem 5, since Bergman function in the adjoint space coincides with function (17) in some angle in the complex plane $\tau(\lambda) \asymp \sqrt{|\lambda|}$ (cf. Lemma 7 in [7]).

It was shown in work [14] that if the boundary of a domain contains an arc so that the curvature in its points is separated from zero and infinity by some constants, then there exist no unconditional bases of exponentials in Smirnov space. This theorem can be obtained from Theorem 5 in the same way.

C) Let $I$ be a bounded interval on the real axis, $h(t)$ be a convex function defined on this interval and $L^{2}(I, h)$ be the space of locally integrable functions on $I$ satisfying the condition

$$
\|f\|:=\sqrt{\int_{I}|f(t)|^{2} e^{-2 h(t)} d t}<\infty .
$$

In works [15], [16], [17], space $\widehat{L}^{2}(I, h)$ was described. It was proven that space $\widehat{L}^{2}(I, h)$ is isomorphic (as a Banach space) to the space of entire functions $F$ obeying the conditions

$$
\begin{gathered}
|F(z)| \leq C_{F} \sqrt{K(z)}, z \in \mathbb{C} . \\
\|F\|^{2}=\int_{\mathbb{R}} \int_{\mathbb{R}} \frac{|F(x+i y)|^{2}}{K(x)} d \widetilde{h}^{\prime}(x) d y<\infty,
\end{gathered}
$$

where

$$
\begin{aligned}
K(z) & =\int_{I}\left|e^{2 z t}\right| e^{-2 h(t)} d t, \\
\widetilde{h}(x) & =\sup _{t \in I}(x t-h(t)) .
\end{aligned}
$$

Thus, the problem on unconditional bases of exponentials in space $L^{2}(I, h)$ becomes equivalent to the problem on unconditional bases of reproducing kernels in the adjoint space of entire functions $\widehat{L}^{2}(I, h)$ and Bergman function is determined there by formula (18). Thus, Theorems 4 or Theorem 5 can be applied as a test for the absence of unconditional bases of exponentials in space $L^{2}(I, h)$. 


\section{BIBLIOGRAPHY}

1. N. Aronszajn Theory of reproducing kernels // Trans. Amer. Math. Soc. 1950. V. 68, No 3. P. 337404.

2. N.K. Nikolski, B.S. Pavlov, S.V. Khruschev. Unconditional bases of exponentials and reproducing kernels, I // Preprint of LDMI. P. 8-80. (in Russian).

3. I.C. Gohberg, M.G. KreĬn. Introduction to the Theory of Linear Nonselfadjoint Operators Moscow: Nauks, 1965. [Amer. Math. Soc., Providence, R.I. 1969.]

4. N.K. Nikolski. Treatise on the shift operator. Moscow: Nauka, 1980. [Springer-Verlag, Berlin. 1986.]

5. K. Seip. Density theorems for sampling and interpolation in the Bargmann-Fock space, I // Reine Angew. Math. 1992. V. 1992. No. 429. P. 91-106.

6. K. Seip, R. Wallsten Density theorems for sampling and interpolation in the Bargmann-Fock space, II // Reine Angew. Math. 1992. V. 1992. No. 429. P. 107-113.

7. K.P. Isaev, R.S. Yulmukhametov. The absence of unconditional bases of exponentials in Bergman spaces on non-polygonal domains // Izv. RAN. Ser. matem. 2007. V. 71, No 6. P. 69-90. [Izv. Math. 2007. V. 71, No 6. P. 1145-1166.]

8. A. Borichev, R. Dhuez, K. Kellay Sampling and interpolation in large Bergman and Fock spaces // J. Funct. Anal. 2007. V. 242, No. 2. P. 563-606.

9. A. Borichev, Yu. Lyubarskii. Riesz bases of reproducing kernels in Fock type spaces // Journal of the Institute of Mathematics of Jussieu. 2010. V. 9. P. 449-461.

10. R.S. Yulmukhametov. Asymptotic approximation of subharmonic functions // Sibir. matem. zhur. 1985. V. 26, No. 4. P. 159-175. [Siberian Math. J. 1986. V. 26, No. 4. P. 603-618.]

11. N.S. Landkof. Foundations of modern potential theory. Moscow: Nauka, 1966. [Springer-Verlag, Berlin. 1972]

12. V.I. Lutsenko, R.S. Yulmukhametov. A generalization of Wiener-Paley theorem to functionals in Smirnov spaces // Trudy MIAN. 1991. V. 200. P. 245-254. [Proc. Steklov Institute of Mathematics. 1993. V. 200. P. 271-280.]

13. K.P. Isaev, R.S. Yulmukhametov. Laplace transforms of functionals on Bergman spaces // Izv. RAN. Ser. matem. 2004. V. 68, No. 1. P. 5-42. [Izv. Math. 2004. V. 68, No. 1. P. 3-41.]

14. V.I. Lutsenko. Unconditional bases of exponentials in Smirnov space. PhD thesis. Institute of Mathematics USC RAS. 1992. (in Russian).

15. V.I. Lutsenko, R.S. Yulmukhametov. Generalization of the Paley-Wiener theorem in weighted spaces // Matem. zam. 1990. V. 48, No. 5. P. 80-87. [Math. Notes Acad. Sci. USSR. 1990. V. 48, No. 5. P. 1131-1136.]

16. V.I. Lutsenko. Paley-Wiener theorem on unbounded interval // Studies on approximation theory. Ufa, 1989. P. 79-85. (in Russian).

17. V.V. Napalkov, R.A. Bashmakov, R.S. Yulmukhametov. Asymptotic behavior of Laplace integrals and geometric characteristics of convex functions // Doklady AN. 2007. V. 413, No. 1. P. 20-22. [Doklady Math. 2007. V. 75, No. 2. P. 190-192.]

18. K.P. Isaev, R.S. Yulmukhametov. Unconditional exponential bases in Hilbert spaces // Ufimskij matem. zhur. 2011. V. 3, No. 1. P. 3-15. [Ufa Math. J. 2011. V. 3, No. 1. P. 3-15.]

Konstantin Petrovich Isaev,

Institute of Mathematics USC RAS,

Chernyshevsky str., 112,

450008, Ufa, Russia

E-mail: orbit81@list.ru

Rinad Salavatovich Yulmukhametov, Institute of Mathematics USC RAS,

Chernyshevsky str., 112,

450008, Ufa, Russia

E-mail: Yulmukhametov@mail.ru 